\title{
Buffering excess hydrogen ions increases ischemic tolerance in hypertrophied myocardium
}

\author{
Koh Takeuchi, MD
}

\author{
From the Division of Cardiovascular Surgery, Yoichi Hospital, Hokkaido, Japan \\ Disclosures: Author has nothing to disclose with regard to commercial support. \\ Received for publication Oct 9, 2018; accepted for publication Oct 9, 2018. \\ Address for reprints: Koh Takeuchi, MD, Division of Cardiovascular Surgery, Yoichi Hospital, 19-1-1 Kurowacho \\ Yoichi, Hokkaido 046-0003, Japan (E-mail: koutakeuchi-circ@umin.ac.jp). \\ J Thorac Cardiovasc Surg 2019;157:1193 \\ 0022-5223/\$36.00 \\ Copyright (c) 2018 by The American Association for Thoracic Surgery \\ https://doi.org/10.1016/j.jtcvs.2018.10.033
}

Cardioplegia is central to myocardial protection in cardiac surgery. Which type of cardioplegia solution is superior has long been discussed. However, there is no general agreement and none became popular in the marketplace. del Nido cardioplegia solution was originally introduced as a myocardial tissue preservation solution, ${ }^{1}$ as was Bretschneider histidine-tryptophan-ketoglutarate (HTK) crystalloid solution. Both have been used for pediatric and adult cardiac surgery. Originally, del Nido solution contained histidine for buffering, but this has been replaced by bicarbonate. Blood also has a strong buffering capacity because it has a high concentration of carbonic anhydrase. In addition, blood has a high oxygen delivery capacity, which may produce more energy during ischemia. Lidocane, which is a sodium channel blocker preventing sodium and calcium accumulation within a cell, stabilizes cell membranes. ${ }^{2}$ Maintaining ionic balance inside cells is probably critical for avoiding ischemia and reperfusion injury. Consequently, we theoretically understand that del Nido cardioplegia solution has many advantages over the other forms of cardioplegia solution, but there is an absence of randomized clinical trials to show the superiority of this cardioplegia-inducing solution.

Talwar and colleagues ${ }^{3}$ conducted a randomized controlled clinical trial comparing del Nido solution and Bretschneider HTK solution in tetralogy of Fallot patients. However, this study has limitations. One cardioplegia system used was blood, and the other was crystalloid solution. This created a locally asanguineous (myocardium) and systemically thin blood condition in 1 group, and locally sanguineous and systemically thick blood in the other group. In fact, their data showed higher hematocrit levels in patients treated with del Nido than Bretschneider HTK during the surgery. Nearly twice as much Bretschneider HTK solution as del Nido solution was infused in almost same amount of time. This means nearly twice as much pressure on the endothelium might occur with the use of Bretschneider HTK solution with potential for endothelial damage. The authors also used different temperature techniques. Temperature, by itself is a mechanism associated with myocardium injury. ${ }^{4}$ Because we cannot obtain

\section{References}

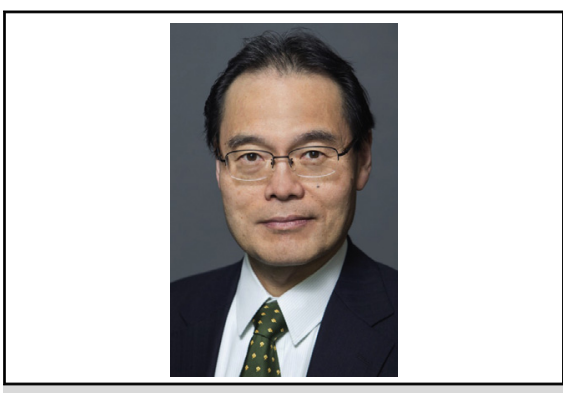

Koh Takeuchi, MD

Central Message

del Nido cardioplegia solution can provide excellent myocardial protection in TOF patients during ischemia. This randomized study warrants an exploration of the advantage of this cardioplegia solution.

See Article page 1182.

preischemic tissue, we are not sure if this solely happened during ischemia. Even with this limitation, this clinical trial was controlled and randomized, so the patients' backgrounds were standarized.

The results of this study show that cardioplegia using del Nido solution leads to quicker cardiac arrest, less ventricular arrhythmia, and better cardiac function. This environment leads to less mechanical ventilation, a shorter intensive care unit stay, and shorter hospital stay. These clinical benefits could result in less edema, less microfibrillar disarray, and higher glycogen storage.

del Nido cardioplegia solution has been popular throughout the world due to its theoretical advantages and ease of use. The results of the clinical study by Talwar and colleagues ${ }^{3}$ support the advantages associated with this cardioplegia solution.

1. Ohkado A, Cao-Danh H, Sommers KE, del Nido PJ. Evaluation of highly buffered lowcalcium solution for long-term preservation of the heart. Comparison with University of Wisconsin solution. J Thorac Cardiovasc Surg. 1994;108:762-71.

2. Matte GS, del Nido PJ. History and use of del Nido cardioplegia solution at Boston Children's Hospital. J Extra Corpor Technol. 2012;44:98-103. Erratum in: J Extra Corpor Technol. 2013;45:262.

3. Talwar S, Chatterjee S, Sreenivas V, Makhija N, Kapoor PM, Choudhary SK, Airan B. Comparison of del Nido and histidine-tryptophan-ketoglutarate cardioplegia solutions in pediatric patients undergoing open heart surgery: a prospective randomized clinical trial. J Thorac Cardiovasc Surg. 2019;157:1182-92.

4. Aguirre VJ, Sinha P, Zimmet A, Lee GA, Kwa L, Rosenfeldt F. Phrenic nerve injury during cardiac surgery: mechanisms, management and prevention. Heart Lung Circ. 2013;22:895-902. 